\title{
Performance Analysis of OFDM Synchronization Using Customized Floating Point for Low Complexity
}

\author{
V. Janakiraman ${ }^{1,2}$, M. Kannan ${ }^{2}$ \\ ${ }^{1}$ Department of Electronics and Communication Engineering, Apollo Engineering College, Chennai, India \\ ${ }^{2}$ Department of Electronics Engineering, MIT, Anna University, Chennai, India \\ Email: janakiraman.g.v@gmail.com,mkannan@mitindia.edu
}

Received 6 May 2016; accepted 16 May 2016; published 19 August 2016

Copyright (C) 2016 by authors and Scientific Research Publishing Inc.

This work is licensed under the Creative Commons Attribution International License (CC BY).

http://creativecommons.org/licenses/by/4.0/

c) (i) Open Access

\begin{abstract}
Orthogonal frequency-division multiplexing (OFDM) is a multi carrier modulation scheme mainly used for digital communications. The performance of OFDM system heavily depends on the synchronization scheme used. In most cases, the accuracy level of synchronization will be worsened by the error caused in fixed point arithmetic involved. In this paper, we analyze the impact of the fixed point arithmetic on the performance of the coarse timing and frequency synchronization. Here with an analytical approach through numerical simulations bit length of IEEE 754 standard single precision format is optimized according to the required degree of accuracy for low complexity. Also, a complete precision level requirement for FFT computations with all possible modulation types is obtained. The proposed precision model is compared with IEEE standard single precision model and its efficiency in OFDM synchronization process is proved through MATLAB simulations. Finally, the complexity reduction of proposed precision model in both addition and subtraction is proved against single precision format using hardware synthesis. Here we proved that more than $\mathbf{5 0 \%}$ complexity reduction is achieved as compared to standard precision models without compromising quality. The quality retention of proposed model is proved in both timing and frequency synchronization process.
\end{abstract}

\section{Keywords}

OFDM, Fast Fourier Transform, Floating Point Aithmetic, Synchronization, Customization

\section{Introduction}

In recent years, floating-point computation has major impact in applications like industrial automation, imaging

How to cite this paper: Janakiraman, V. and Kannan, M. (2016) Performance Analysis of OFDM Synchronization Using Customized Floating Point for Low Complexity. Circuits and Systems, 7, 3112-3120. http://dx.doi.org/10.4236/cs.2016.710264 
processing, and signal computing where high dynamic range is an essential. But due to arithmetic complexity of using floating-point computation still fixed-point computation is more common in many applications. FFT is an efficient computation of the DFT, which provides a sampled Fourier Transform, for discrete-time finite length sequences.

Though FFT is extensively used to analyze the signal characteristics, it is now a basic building block in any broadband wireless communications used for sub carrier mapping. OFDM is the most prominent method in any wireless standards because of its robustness against linear time variant channel conditions. OFDM is robust against multipath fading still it has some significant challenges due to its sensitiveness to synchronization errors; it makes the task of estimating these errors for proper synchronization. In many papers, they were used pre defined binary sequences for correcting both timing and frequency offsets P. H. Moose, J.-J. van de Beek and H. Minn et al. [1]-[3]. But they used only fixed points arithmetic's where both truncation and quantization errors are major concern for high accuracy. In recent years, needs of high performance applications like multimedia, wireless teleconferencing leads to use various range of data modulations like BPSK, QPSK, 16-QAM, and 64QAM were used T. M. Schmidl and H.-K. Song et al. [4] [5]. Even in recent advanced TDS-OFDM maximum constellation used is 256-QAM for digital terrestrial video broadcasting due to its high data rate requirements. During data modulation input digital data input will be converted into complex signals which will be mapped on channel using IFFT block. In most cases 32-bit complex symbols were used where 16-bit for real part and 16-bit for imaginary part each will cover dynamic range of $2^{-16}$ to $2^{16}$ with considerable quantization error during FFT computations.

This paper starts by introducing synchronization process in OFDM. The effect of arithmetic computation in synchronization and also provides reasons why high precision and low complexity are desirable.

\section{OFDM Synchronization}

\subsection{Preamble}

The baseband modulated signal of OFDM systems is transmitted across various sub bands by frequency hopping. A symbol is a concatenation of IFFT output of samples plus CP. Dedicated preambles are used for timing synchronization, carrier frequency offset recovery and channel estimation. The 802.11a standard M. Ghogho et al. [6] defines the preamble as shown in Figure 1. Every frame has the preamble. The preamble consists of ten identical short training symbols (t1, t2..., t10) and two identical long training symbols. The preamble is converted into a real baseband signal using IFFT computation as shown in Figure 1.

\subsection{Timing Synchronization}

Timing synchronization is achieved by using a training sequence whose first half is equal to its second half in the time domain. In receiver side both auto correlation in Equation (1) and cross-correlation in Equation (2) techniques are used for fine time synchronization as shown in Figure 2. However, this requires significant resources several multipliers are used Hamed Abdzadeh et al. [7]. From the peak value of cross correlation function frame detection is carried out. Although the peak occurs exactly at the correct timing, we found that its peak
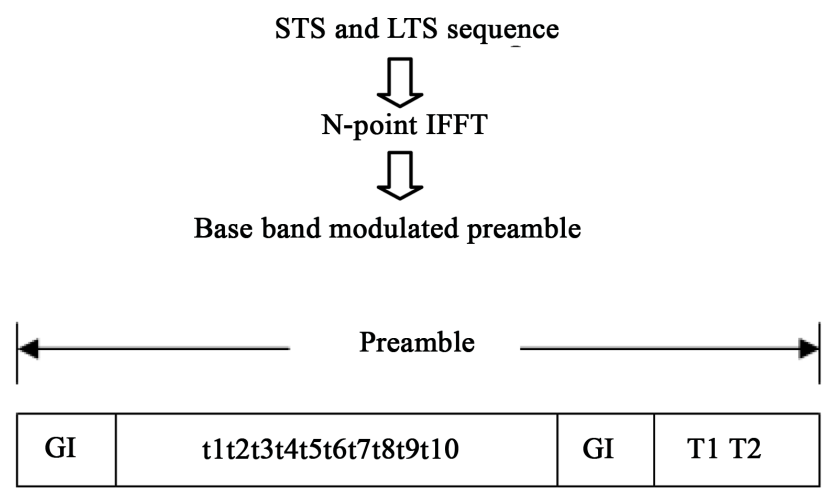

Figure 1. IEEE 802.11a preamble. 


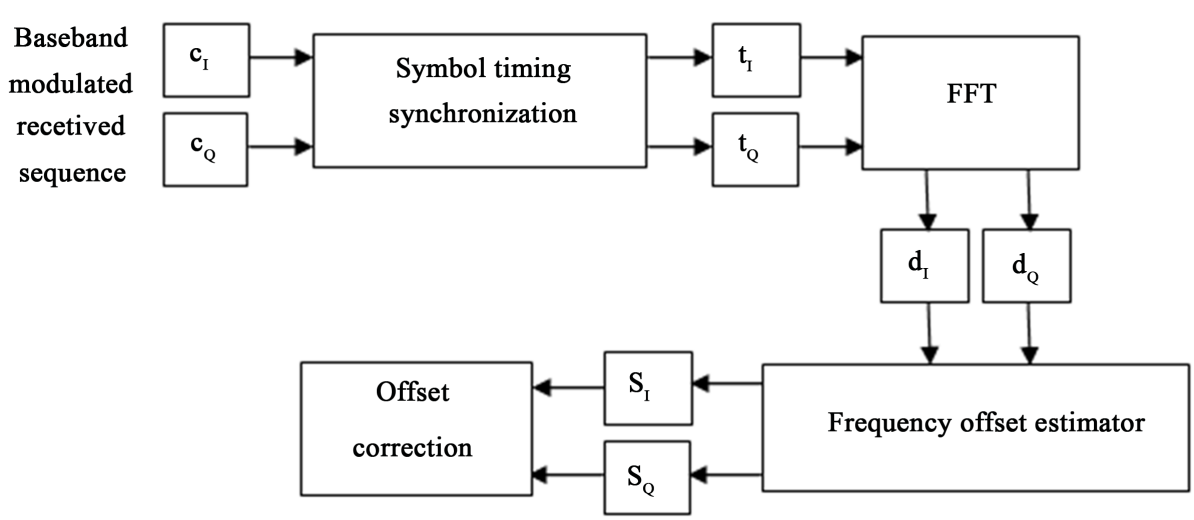

Figure 2. Timing and frequency offset estimator architecture.

magnitude is very sensitive to AWGN and multi-path channels, which makes the estimation over a threshold difficult.

$$
Y(n)=\operatorname{yrLTS}(n) * y \operatorname{LTS}(-n)
$$

where * is the convolution operator, $y r L T S$ is the received sequence and $y(n)$ is the output.

$$
C k=\sum_{n=0}^{L-1} r k-m * X m
$$

where $L$ is the length of the cross-correlation.

\subsection{Frequency Synchronization}

Since OFDM system is very sensitive to frequency offset, high precision in frequency offset estimation and compensation is important Zoran Cvetkovic et al. [8]. This will makes us to use high precision computation than fixed point processing where high precision is not possible. In the presence of frequency offset the received sequence has the form as shown in Equation (3).

$$
r m=\mathrm{e}^{j 2 \pi f \in n} \sum_{n=0}^{L-1} s m-n h n+w n, 0 \leq m \leq N-1
$$

\section{FFT Arithmetic Computation}

\subsection{Fixed vs. Floating-Point}

During the course of an FFT computation it is well known that to avoid loss of dynamic range, numerical issues much be dealt with at each butterfly computation stage, leading to a variety of tradeoffs. For example in many cases 32 bits are used to represent complex symbols to avoid loss of coverage during IFFT computations on transmitter side. In best case maximum constellation order used is 64-QAM with maximum possible FFT length will be 2048 point.

Fixed point design was based on following parameters:

- Dynamic range of each signal involved.

- Minimum number of bits required for it.

- Robustness analyzes by performing BER simulations.

\subsection{Analyzes of Dynamic Ranges}

In real time processing to minimize hardware resources, the word length is kept same by rounding results after appropriate groups of operations, but in this case dynamic range would suffer. Alternatively, word lengths could grow a fixed number of bits after each arithmetic operation to accommodate final results. Here through exhaustive MATLAB simulations dynamic ranges of all modulation types are calculated and showed in Table 1 . Behavior of overall system performance with different modulation scheme is also verified through MATLAB simulation as shown in Figure 3. 


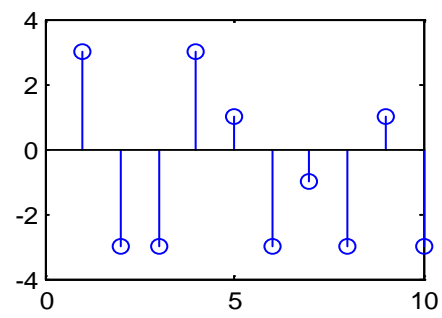

(a)

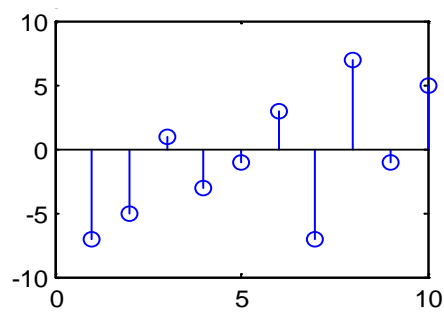

(c)

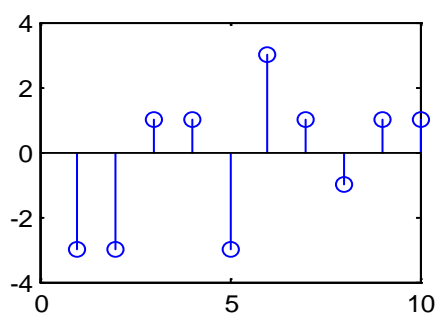

(b)

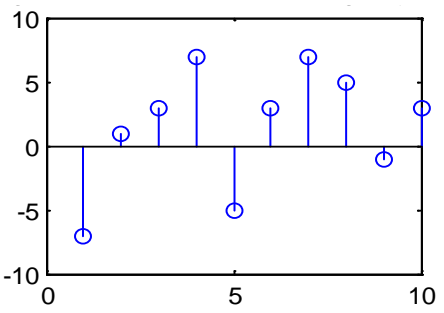

(d)

Figure 3. Dyanmaic range of all possible constellation points of different modulation. (a) 16 QAM modulation (Real part); (b) 16 QAM modulation (imaginary part); (c) 64 QAM modulation (Real part); (b) 64 QAM modulation (imaginary part).

Table 1. Dynamic range of all possible moduation types.

\begin{tabular}{ccc}
\hline Modulation type & Maximum constellation output & Maximum possible value from 2018 FFT \\
\hline BPSK (2) & $1+0 \mathrm{j}$ or $0+1 \mathrm{j}$ & 2048 \\
QPSK (4) & $1+1 \mathrm{j}$ & $2.048 \mathrm{e}+003+2.048 \mathrm{e}+003 \mathrm{j}$ \\
16-QAM (16) & $3+3 \mathrm{j}$ & $6.144 \mathrm{e}+003+6.144 \mathrm{e}+003 \mathrm{j}$ \\
64-QAM (64) & $7+7 \mathrm{j}$ & $1.4336 \mathrm{e}+004+1.4336 \mathrm{e}+004 \mathrm{j}$ \\
\hline
\end{tabular}

\subsection{Effects of Precision in OFDM Synchronization}

In recent years many papers have been published on the effect of time and frequency offset on the performance of an OFDM system, as in F. Tufvesson, E. G. Larsson et al. [9]-[11]. However, for software designs these methods using high-precision FP operations, to analyze actual behavior of system. But the implementation of entire OFDM system with floating point computation is not possible one because of its high hardware complexity. For IC fabrication designers ultimately implement any complex algorithms in integer-like hardware, that is, fixed-point units F. J. Lopez-Martinez et al. [12]. In order to meet the real-time processing requirements many multipliers less methods were introduced where, they used only shifters and adders K.-W. Yip and Thinh H. Pham et al. [13] [14]. But the use of fixed point arithmetic to implement the system in which fraction numbers are involved will cause some perceptible error.

However, in fixed point truncation of LSB part to keep output bit width as constant leads rounding error and fraction numbers are not represented cause quantization error. It leads to some considerable degradation in signal precision. Many error compensation algorithms have been proposed still all are insufficient when quality is demanded. In any OFDM system synchronization is done by sending some identical training symbols repeatedly. In receiver side cross correlator uses these training symbols as a known reference signal and the timing and frequency offset can be easily estimated using log-likelihood function Michele Morelli et al. [15].

Low-power and area efficient on-chip design models only preferred. High accuracy precision model is required for accurate estimation channels time varying nature. By considering all these factors into account, here we proposed an effective implementation of the synchronization algorithms with least precision FP model. Here word length optimization is performed in standard FP computations to improve the design performance with reduced complexity. 


\subsection{Customizable Floating Point Format}

In FP arithmetic dynamic range is determined by exponent bit-width. We found the maximum possible dynamic range of various modulation types with 2048 point FFT in Table 2.

On the other hand, the accuracy of FFT is not degraded when narrowing the bit-width of exponent and mantissa. The influence of decreasing bit-width on standard IEEE single precision 32-bit floating point will lead the way to use floating point arithmetic based FFT computations in all wireless applications. In order to find the basic exponent bit-width required to cover the values given in Table 2, we derive the dynamic range of modified FP unit with 10 bit mantissa and 5 exponent bit width.

\section{Results and Analysis}

\subsection{Simulation Results and Analysis}

In this section, the performance of proposed model is compared with fixed point and high precision model by simulating it on MATLAB platform. In our simulation, 64 sub-carriers, 16-QAM modulation are used, CP is chosen as N/4 N, the simulation is based on AWGN channel, and for synchronization standard 802.11a preamble is used.

From Figure 4, we can see the abs error difference proposed method is obviously much lower than fixed point for both STS and LTS sequence and the scope changes in error value in proposed method is much smaller than fixed point method. It explains that the proposed method performs well and has better robustness over dynamic range and precision. Here we showed that the error level of fixed point computation is quite high in LTS sequence over STS due to its complex structures which will cause major problem during OFDM synchronizing process.

However, the performance degradation of fixed point model will always be greater than timing synchronization process because of its FFT computation on receiver side. Due to stage wise computation of FFT error caused at one stage will propagate through all other remaining stages leads severe performance degradation during channel estimation process. Therefore, the performance loss of fixed point FFT computation should be taken into account when frequency offset is computed. In our proposed customized model there is no performance loss during FFT computation as it can cover all dynamic ranges as we discussed earlier (Figure 5).

From Figure 6 the impulse of cross-correlation peak has a good timing synchronization performance in both single precision and proposed customized precision models. But with considerable frequency offset with noisy environment the performance fixed point cross-correlation end with three peaks leads misdetection of frames. In most cases the possibility of misdetection is very high in fixed point arithmetic due to quantization effects. In our proposed model we were used 10 bits for mantissa which is enough to avoid misdetection during timing synchronization process.

\subsection{VLSI Implementation Results}

The complexity of the synchronization scheme largely depends on bit-width used to represent the symbols. In this paper, we compare our proposed precision model with high precision for complexity reduction. The proposed architecture is modeled using the Verilog HDL and synthesized using ALTERA Cyclone III EP3C16F484C6 device. Detailed area results are shown in Table 3 and Table 4, and the results show proposed model reduce complexity more than $50 \%$ and $1 / 3^{\text {rd }}$ for addition and multiplication respectively as compared to single precision model. The design saves more area in time synchronization since large number of addition and multiplications are used in correlators.

\section{Conclusion}

Here we proved the bit reduction efficiency of floating point computation and its robustness against computa-

Table 2. Dynamic range of modified FP unit.

\begin{tabular}{cccccc}
\hline Customized FP & Sign & Exponent & Mantissa & Min \\
\hline 16 bit & 1 bit & 5 bit & 10 bit & $2.9802 e^{-008}$ & $6.5536 e^{+004}$ \\
\hline
\end{tabular}




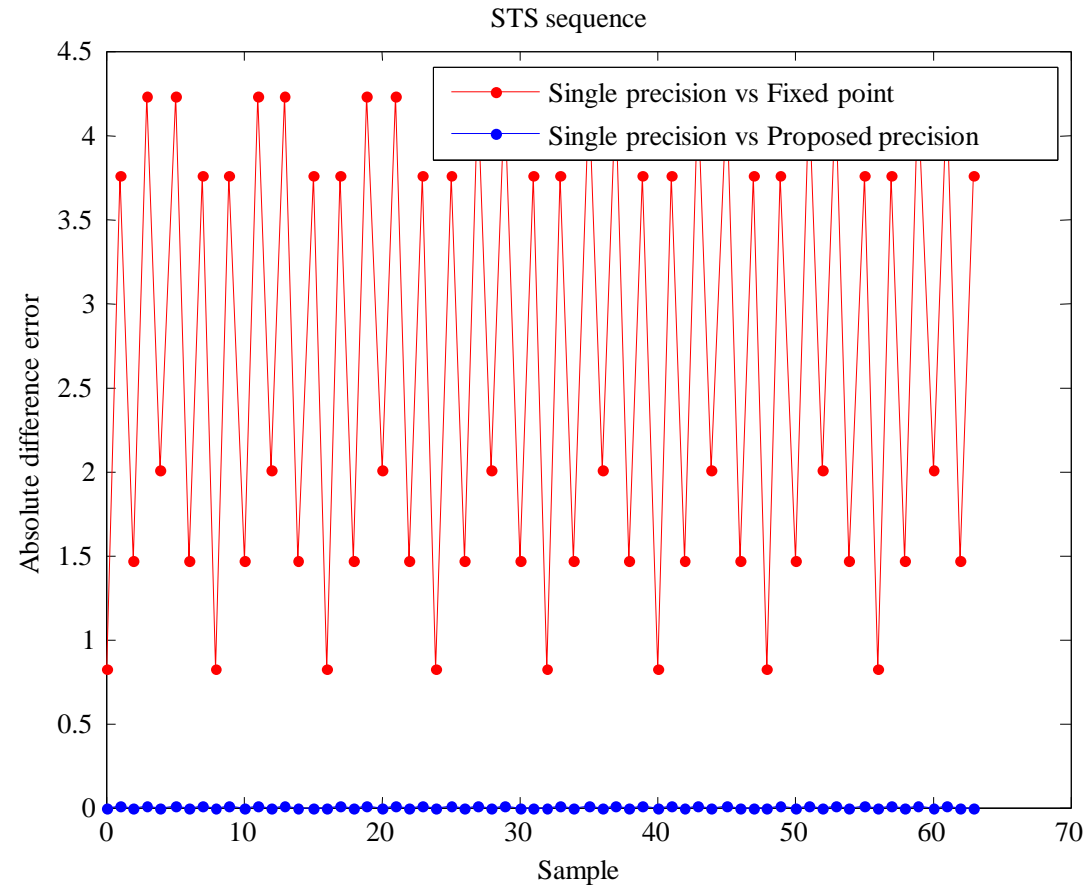

(a)

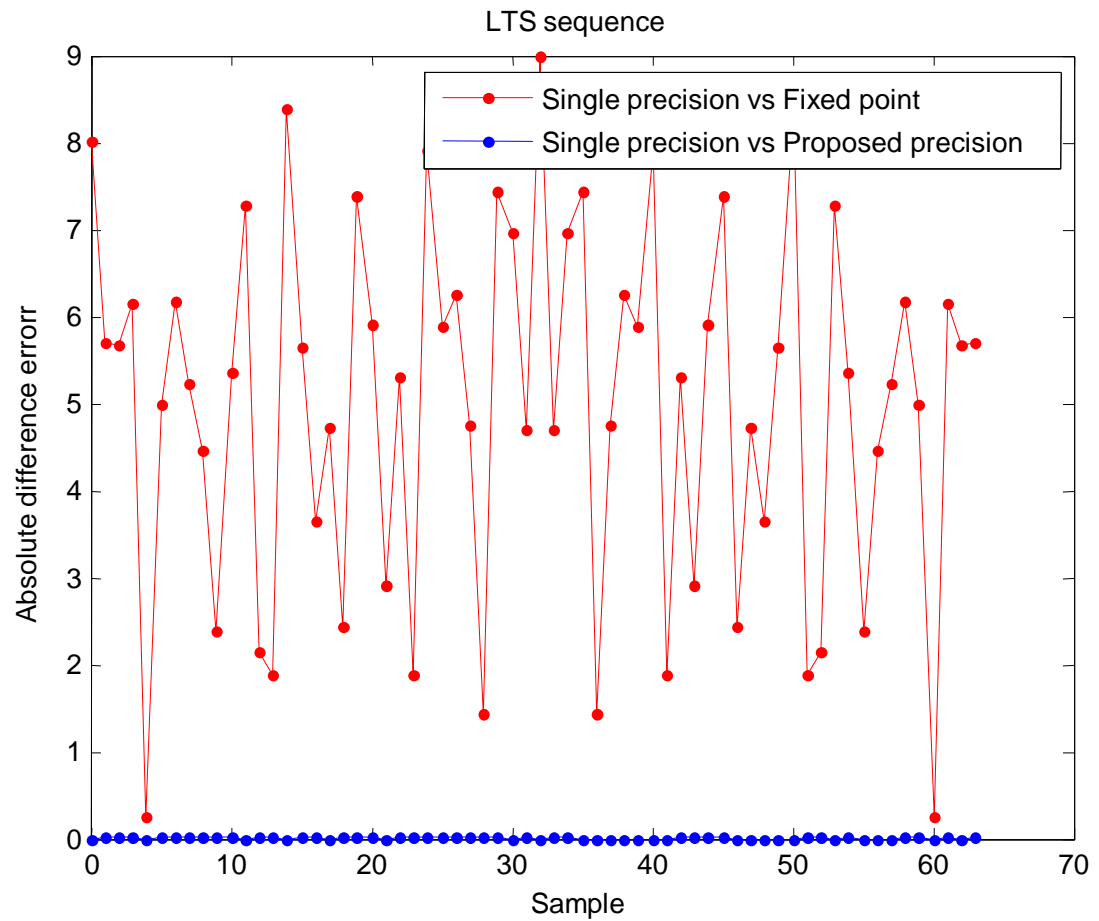

(b)

Figure 4. (a) Absolute error difference of STS sequence; (b) Absolute error difference of LTS sequence.

Table 3. Synthesize reports of addition.

\begin{tabular}{cccc}
\hline Logic utilization & Single precision & Fixed point & Customized floating point \\
\hline Logical elements & 347 & 49 & 154 \\
\hline
\end{tabular}




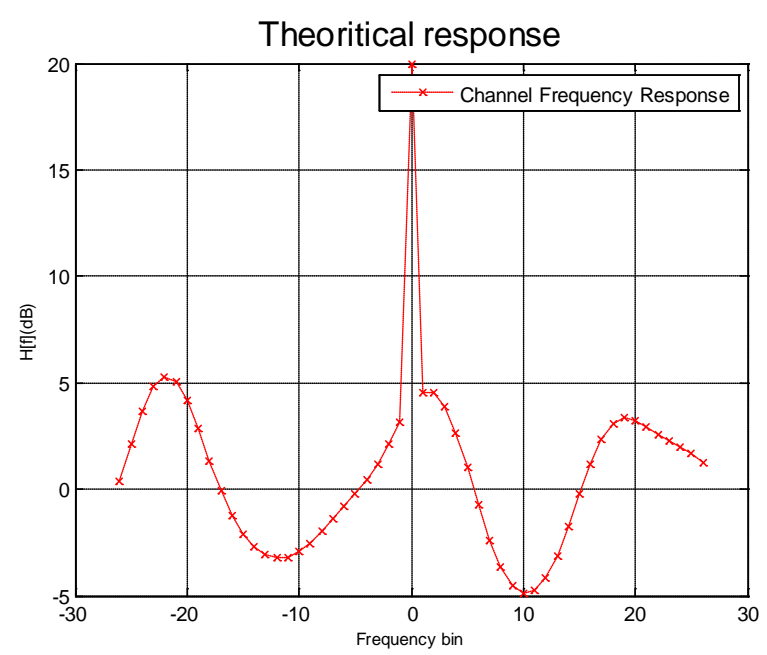

(a)

Estimated channel response with fixed point

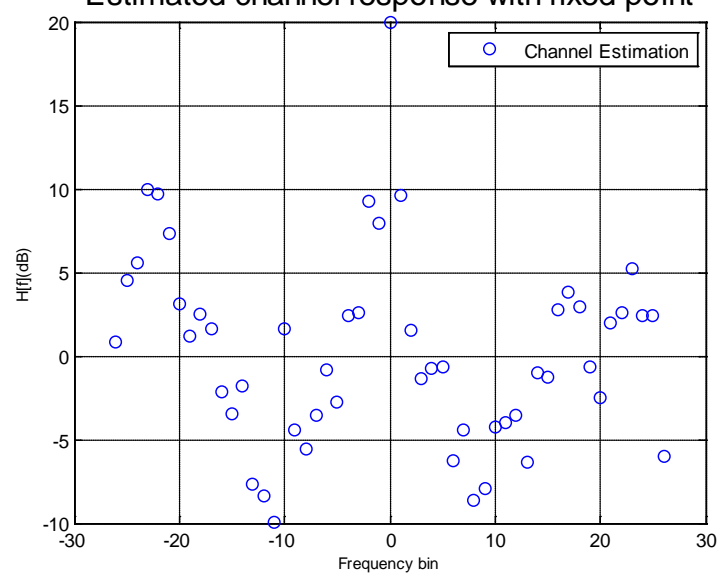

(c)
Estimated channel response with full precision

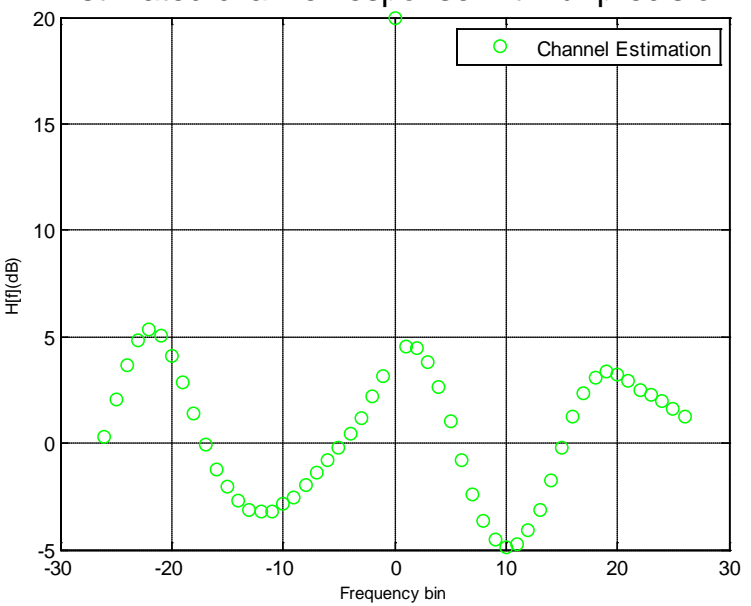

(b)

Estimated channel response with proposed precision

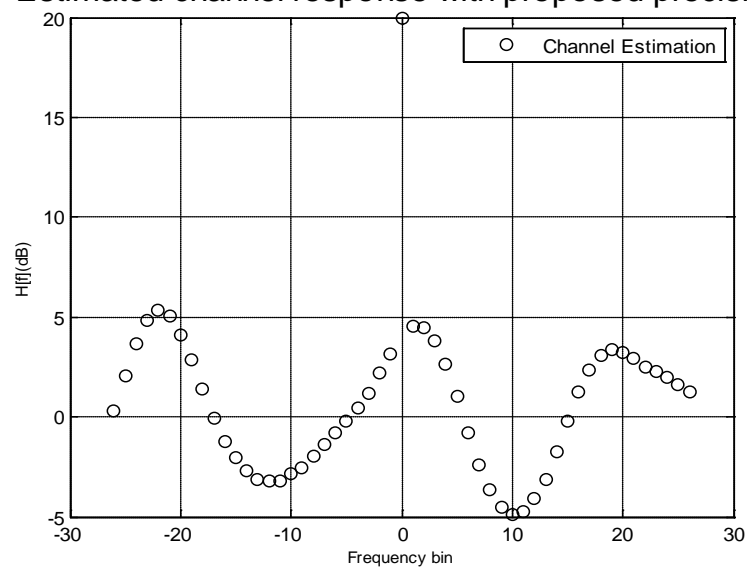

(d)

Figure 5. (a) Theoritical Response of channel frequency; (b) Estimated channel Response with full precision; Estimated Channel Response with fixed point; (d) Estimated channel Response with proposed precision.

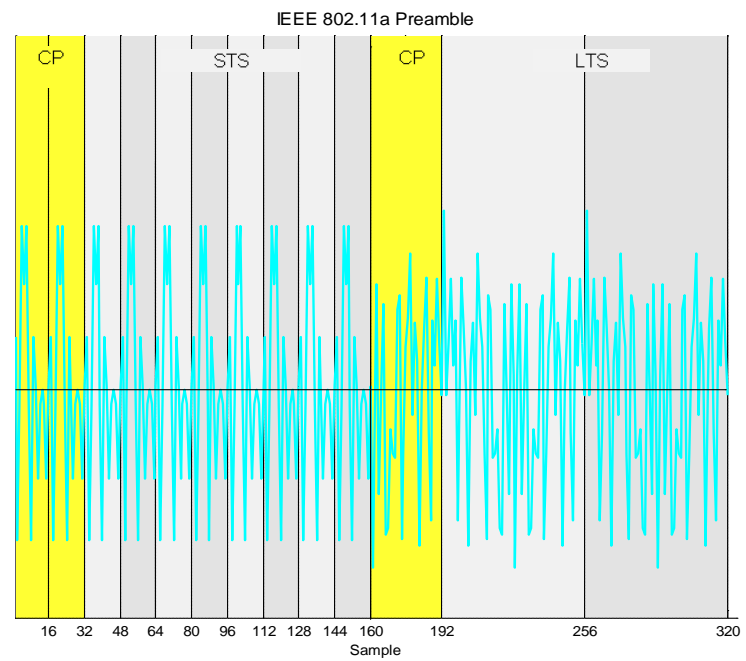

(a)

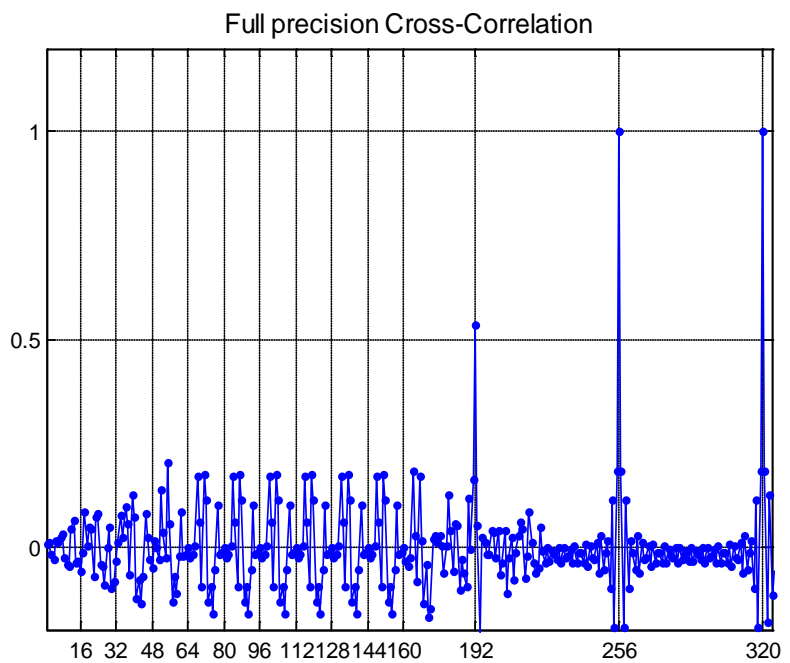

(b) 


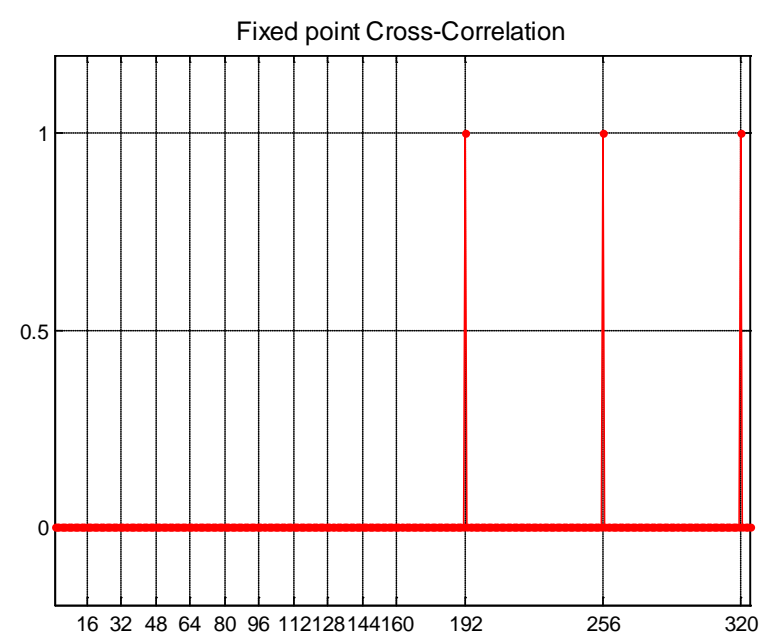

(c)

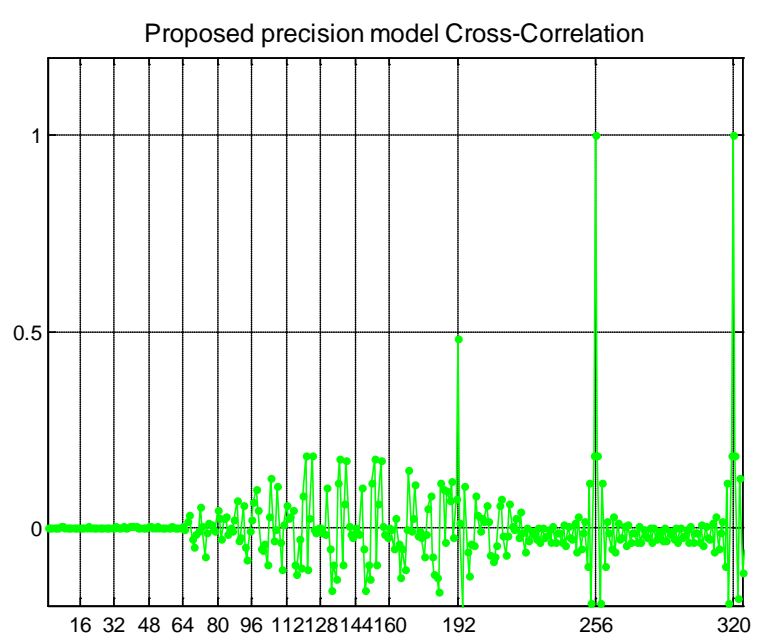

(d)

Figure 6. (a) IEEE802.11 a preamble; (b) Full Precision cross-correlation; (c) Fixed point cross correlation; (d) Proposed precision model cross-correlation.

Table 4. Synthesize reports of multiplication.

\begin{tabular}{cccc}
\hline Logic utilization & Single precision & Fixed point & customized floating point \\
\hline Logical elements & 97 & 49 & 27 \\
9-bit embedded multiplier & 7 & 2 & 2 \\
\hline
\end{tabular}

tional errors. The OFDM system is very sensitive to frequency offset, high precision in frequency offset computation is important for an accurate compensation. The proposed precision can cover all dynamic ranges and its efficiency and complexity over full precision model is verified. The results show that the developed customized floating point based OFDM can consume very less number of resources to provide cost effective and high performance solution as compared to single precision models for wireless communication applications. It is also observed that there is no performance gab between full precision and proposed precision model in both time and frequency synchronization. To extend this proposed model for low complexity, one can reduce the mantissa bit sizes by compromising the precision level of OFDM system model.

\section{Acknowledgements}

The author gratefully acknowledges the support provided by his supervisor Dr. M. Kannan, Mr. D. Rajaram and thank the management of Apollo Engineering College for encouraging the research work.

\section{References}

[1] Moose, P.H. (1994) A Technique for Orthogonal Frequency Division Multiplexing Frequency Offset Correction. IEEE Transactions on Communications, 42, 2908-2914. http://dx.doi.org/10.1109/26.328961

[2] van de Beek, J.-J., Sandell, M. and Borjesson, P.O. (1997) ML Estimation of Time and Frequency Offset in OFDM Systems. IEEE Transactions on Signal Processing, 45, 1800-1805. http://dx.doi.org/10.1109/78.599949

[3] Minn, H., Bhargava, V.K. and Letaief, K.B. (2003) A Robust Timing and Frequency Synchronization for OFDM Systems. IEEE Transactions on Wireless Communications, 2, 822-839. http://dx.doi.org/10.1109/TWC.2003.814346

[4] Schmidl, T.M. and Cox, D.C. (1997) Robust Frequency and Timing Synchronization for OFDM. IEEE Transactions on Communications, 45, 1613-1621. http://dx.doi.org/10.1109/26.650240

[5] Song, H.-K., You, Y.-H., Paik, J.-H. and Cho, Y.-S. (2000) Frequency Offset Synchronization and Channel Estimation for OFDM-Based Transmission. IEEE Communications Letters, 4, 95-97. http://dx.doi.org/10.1109/4234.831036

[6] Ghogho, M. and Swami, A. (2008) Frame and Frequency Acquisition for OFDM. IEEE Signal Processing Letters, 15, 605-608. http://dx.doi.org/10.1109/LSP.2008.2001806 
[7] Hamed Abdzadeh, Z. and Shayesteh, M.G. (2012) A Novel Preamble-Based Frame Timing Estimator for OFDM Systems. IEEE Communications Letters, 16.

[8] Cvetkovic, Z., Tarokh, V. and Yoon, S. (2013) On Frequency Offset Estimation for OFDM. IEEE Transactions on Wireless Communications, 12.

[9] Tufvesson, F., Edfors, O. and Faulkner, M. (1999) Time and Frequency Synchronization for OFDM Using PN-Sequence Preambles. Proceedings of the IEEE VTC, 2203-2207.

[10] Yip, K.W., Wu, Y.C. and Ng, T.S. (2004) Timing-Synchronization Analysis for IEEE802.11a Wireless LANs in Frequency-Nonselective Rician Fading Environments. IEEE Transactions on Wireless Communications, 3, 387-394. http://dx.doi.org/10.1109/TWC.2004.825372

[11] Larsson, E.G., Liu, G., Li, J. and Giannakis, G.B. (2001) Joint Symbol Timing and Channel Estimation for OFDM Based WLANs. IEEE Communications Letters, 5, 325-327. http://dx.doi.org/10.1109/4234.940980

[12] Lopez-Martinez, F.J., Martos-Naya, E., Entrambasaguas, J.T. and Garcia-Abril, M. (2007) Low Complexity Synchronization and Frequency Equalization for OFDM Systems. IEEE.

[13] Yip, K.-W., Wu, Y.-C. and Ng, T.-S. (2003) Design of Multiplierless Correlators for Timing Synchronization in IEEE 802.11a Wireless LANs. IEEE Transactions on Consumer Electronics, 49, 107-114. http://dx.doi.org/10.1109/TCE.2003.1205462

[14] Pham, T.H., Fahmy, S.A. and McLoughlin, I.V. (2012) Low-Power Correlation for IEEE 802.16 OFDM Synchronization on FPGA. IEEE Transactions on VLSI Systems.

[15] Morelli, M. and Moretti, M. (2013) Joint Maximum Likelihood Estimation of CFO, Noise Power, and SNR in OFDM Systems. IEEE Wireless Communications Letters, 2.

\section{Submit or recommend next manuscript to SCIRP and we will provide best service for you:}

Accepting pre-submission inquiries through Email, Facebook, LinkedIn, Twitter, etc. A wide selection of journals (inclusive of 9 subjects, more than 200 journals)

Providing 24-hour high-quality service

User-friendly online submission system

Fair and swift peer-review system

Efficient typesetting and proofreading procedure

Display of the result of downloads and visits, as well as the number of cited articles

Maximum dissemination of your research work

Submit your manuscript at: http://papersubmission.scirp.org/ 